\title{
A Logistical Approach to Managing the Resources of Multi Nomenclature Spare Parts of a Corporate Car Service in Conditions of Risk and Uncertainty of Demand
}

\author{
Karimov Nijat Ashraf \\ Department of Automotive Engineering, Azerbaijan Technical University, Baku, Azerbaijan \\ Email address: \\ nicat.kerimov12@gmail.com \\ To cite this article: \\ Karimov Nijat Ashraf. A Logistical Approach to Managing the Resources of Multi Nomenclature Spare Parts of a Corporate Car Service in \\ Conditions of Risk and Uncertainty of Demand. Science Journal of Business and Management. Vol. 5, No. 4, 2017 , pp. 169-174. \\ doi: $10.11648 /$ j.sjbm.20170504.15
}

Received: June 8, 2017; Accepted: July 25, 2017; Published: August 15, 2017

\begin{abstract}
The task of determining the optimal sizes of spare parts for an auto-service enterprise based on the maximum profit criterion for a discrete distribution of demand is formulated as a problem of quadratic programming with linear constraints. To calculate the probabilistic measure of the distribution of the values of the demand vector components, an approximation is used of the empirical distribution function of the demand components by hyper-Erlanger distribution functions, and the subsequent calculation of the corresponding distribution densities.
\end{abstract}

Keywords: Spare Parts, Storage Costs, Costs for Fulfilling Orders, Distribution

\section{Introduction}

In recent years, the concept of logistics has been developed and is being used as an important approach to inventory management [1-8]. Logistics is aimed at reducing costs, increasing reliability, reducing risks through harmonization and mutual systemic adjustment of plans and actions of the supply, production and marketing segments of the enterprise.

Current transformations in the transport sector of the republic are characterized by changes both in the size of the fleet of serviced rolling stock and in the structure of the management of motor transport enterprises (ATP). Unlike the conditions of the planned economy, when the demand for ATP transport services exceeded the capabilities of the motor transport service enterprises and it was possible to realize these opportunities regardless of the ATP composition used, this situation changed radically with the transition to the buyer's market. The task of economical and successful implementation of the capabilities of service centers in a competitive market in the transport services market is becoming one of the main tasks. Necessary conditions for its solution are the rapid reaction of enterprises to changing needs, reducing costs for the production of transport services and improving their quality and reliability.
The most common model of applied logistics theory is the model of the optimal or economical order size (EOQ) for the replenishment period $[7,9,10]$. A review of the EOQ models and their bibliography is given in [11]. The problem of uncertainty and classification of types of uncertainty in supply chains are considered in [12].

In practice, there are often situations where the data on the prehistory of the supply of spare parts is either not sufficiently representative or inaccessible. Then, for inventory management, the demand is modeled on the basis of expert assessments, which contain more subjectivity than chance. In such cases, the inventory management problem is formulated as an optimization problem under fuzzy information [13-17]. In some works (see, for example, [18, 19]) single-period (single-period) control problems of singleand multi-item reserves are solved using the strategy of minimal average and conditional Risk or neutral risk.

In this paper we use the method of neutral risk [19] for the one-time task of managing multinomenclature stocks, in which demand is described by a discrete probability distribution. For constructing a discrete probability distribution, we use the approximation of the empirical distribution function of actual demand in the previous period of replenishment of spare parts of an auto service plant with the help of the hyper-relang 
function of distribution in the Levy metric. The accuracy of the hypererlang approximation of arbitrary distributions in various metrics is estimated in [20].

It is shown that for an arbitrary empirical distribution function and a given degree of accuracy of the approximation $\delta$, one can select the step of discretizing $\delta_{i}$ the values of the random $t_{i}$ demand of the $D_{i} i$ nomenclature of spare parts, in which the entire observation interval $N_{i}$ is divided into subintervals and the hyperErlanger distribution function (weighted sum of Erlanger distributions) corresponding to this partitioning, Approximates the empirical distribution function with a given accuracy $\delta$ in the Levy metric. The geometric meaning of this metric is that its value is equal to the side of the maximum square inscribed between the graphs of the empirical distribution $F(x)$ function and the approximating distribution function $F_{X}(x)$. This approach is universal and can be used to approximate the empirical distribution functions of arbitrary random variables.

The probability $\mu_{i j}$ of the appearance of the values of a $t_{i j}$ discrete random variable $X_{i}=D_{i}$ is equal to the values of the probability density $\mu_{i}(x)$ function at a point $x=t_{i j}$, where it is assumed that $\mu_{i}(x)=\frac{d}{d x} F_{X}(x)$, where $F_{X}(x)$ is the distribution function $X_{i}$.

It should be borne in mind that the problem of approximate calculation of the derivative $\left.\mu_{i}(x)\right|_{x=t_{i, j}}$ value by means of a difference operator $R(u, \alpha)=(u(x+\alpha)-u(x)) / \alpha$, where $u(x)=E h_{X i}(x)\left(E h_{X_{i}}(x)\right.$ is an approximation $F_{X_{i}}(x)$ with accuracy $\delta$ ) is incorrect. $R(u, \alpha)$ will be a regularizing operator in the sense of Tikhonov only for $\alpha=\delta / \eta(\delta)$, where $\eta(\delta) \rightarrow 0$ for. It is enough to choose $\eta(\delta)=\delta^{\varepsilon}, \quad 0<\varepsilon<1$, then $\alpha=\delta^{\varepsilon}$.

The problem of optimal allocation of a multi nomenclature order $x=\left(x_{1}, \ldots, x_{n}\right)$ based on the maximum profit criterion formulated in the paper is reduced to solving the problem of quadratic programming. In particular, with the proportional dependence of the amount of costs on the purchase of the ordered product of the type $i$ on the value $A_{i} / x_{i}\left(A_{i}\right.$ - the need for the ordered product in the period under consideration), maximizing the expected average value of profit leads simultaneously to minimizing the total cost of fulfilling the order for this product, i.e. To the logistic model EOQ.

\section{Formulation of the Optimization Problem and Its Solution}

Let us formulate a one-period multinomenclature problem of managing a single-period multi-item for an auto service enterprise, taking into account two types of costs:

1) Costs for fulfilling orders: $\mathrm{a}_{\mathrm{i}}>0$, equal to the sum of costs for the purchase of the ordered product of type $i$, dollar;
2) Storage costs: the average number of units $i$, which will have to be stored in the warehouse, when ordering the size $x_{i}$ (pieces) is $\mathrm{x}_{\mathrm{i}} / 2$ (pieces).

The amount of storage costs should be proportional to the number of stored product units and storage time $T_{i}=x_{i} / D_{i}$, where $\mathrm{D}_{\mathrm{i}}$ is the fuzzy demand for the product $i$. Then the expected value of storage costs will be $m_{i} \cdot h_{i} \cdot x_{i}^{2} / 2$, where $\mathrm{h}_{\mathrm{i}}$ is the storage cost per unit of product $i$ and

$$
m_{i}=E\left[\frac{1}{D_{i}}\right]=\int_{0}^{\infty} \operatorname{Cr}\left\{\frac{1}{D} \geq r\right\} d r
$$

Here, $\mathrm{Cr}$ is the credibility measure defined in [14].

When determining the optimal size of an order $x_{i}$, using the maximum profit criterion, the expected value of profit is usually used as the objective function. In the case of the single-item inventory management task $i$, the objective function has the form

$$
\pi_{i}\left(x_{i}, D_{i}\right)=p_{i} \cdot x_{i}-a_{i}-\frac{h_{i} \cdot x_{i}^{2}}{2 D_{i}}
$$

Where: $\mathrm{p}_{\mathrm{i}}$ is the purchase price of product order unit $i$, dollar.

The expected value of the fuzzy profit $\pi\left(x_{i}, D_{i}\right)$ is denoted by $E\left[\pi\left(x_{i}, D_{i}\right)\right]$. Using the properties of the operation $\mathrm{E}$, we obtain

$$
E\left[\pi_{i}\left(x_{i}, D_{i}\right)\right]=p_{i} x_{i}-a_{i}-\frac{h_{i} \cdot x_{i}^{2}}{2 D_{i}} E\left[\frac{1}{D_{i}}\right]
$$

Thus, for a one-period one-nomenclature problem, the optimization problem will be written in the form

$$
\left\{\begin{array}{c}
\max E[\pi(x, D)] \\
x \geq 0
\end{array}\right.
$$

where

$$
E[\pi(x, D)]=p \cdot x-a-\frac{h \cdot x^{2}}{2} \cdot m, m=E\left(\frac{1}{D}\right) .
$$

The solution of problem (4) is

$$
x^{*}=\frac{p}{h m}
$$

As an approximate (whole) solution of the problem (4), we assume

$$
\hat{x}^{*}=\operatorname{integ}\left(\frac{p}{h \cdot m}\right)
$$

Where integ is the integer part of a number.

For $a=c_{\text {expent }} \cdot A / x$, where $c_{\text {expent }}$ - the cost of one order, dollar; A - the demand for the ordered product during the given period, pcs., The maximization of the expected value $E[\pi(x, D)]$ leads simultaneously to minimization of the total costs for the ordered product.

For the multinomenclature problem, we assume that there is no connection between any two standard terminals. Under this condition, the profit function is written in the form 


$$
\pi(x, D)=\sum_{i=1}^{n}\left(p_{i} x_{i}-a_{i}-\frac{h_{i} x_{i}^{2}}{2 D_{i}}\right)
$$

Where $x=\left(x_{1}, \ldots, x_{n}\right), D=\left(D_{1}, \ldots, D_{n}\right)$ are vectors from $n$ components. Under the criterion of neutral risk, the multinomenclature problem of inventory management will be written down as an optimization problem

$$
\left\{\begin{array}{c}
\max E[\pi(x, D)] \\
x \geq 0
\end{array}\right.
$$

Where the condition $\mathrm{x} \geq 0$ means $x_{i} \geq 0(i=1, \ldots, n)$.

Suppose that the components $D_{i}$ of the vector $\mathrm{D}$ are mutually independent fuzzy quantities in the sense of the definition of [21], then their joint possible distribution $\mu_{D}$ is represented in the form

$$
\mu_{D}\left(t_{1}, t_{2}, \ldots, t_{n}\right)=\min _{1 \leq i \leq n} \mu_{D_{i}}\left(t_{i}\right)
$$

let

$$
\pi_{i}\left(x_{i}, D_{i}\right)=p_{i} x_{i}-a_{i}-\frac{h_{i} x_{i}^{2}}{2 D_{i}}, i=1,2, \ldots, n .
$$

Then $\pi_{i}\left(x_{i}, D_{i}\right)$ are also mutually independent fuzzy quantities. In view of the linear independence of the operator of the expected value [22], we have

$$
E[\pi(x, D)]=\sum_{i=1}^{n}\left(p_{i} \cdot x_{i}-a_{i}-\frac{h_{i} x_{i}^{2}}{2 D_{i}} E\left[\frac{1}{D_{i}}\right]\right)
$$

Consequently, problem (7) will be equivalent to the following optimization problem

$$
\left\{\begin{array}{c}
\max \sum_{i=1}^{n}\left(p_{i} \cdot x_{i}-a_{i}-\frac{m_{i} h_{i} x_{i}^{2}}{2}\right) \\
x \geq 0
\end{array}\right.
$$

where

$$
m_{i}=E\left[\frac{1}{D_{i}}\right], i=1,2, \ldots, n
$$

Solving equations

$$
\frac{\partial}{\partial x_{i}} \sum_{i=1}^{n}\left(p_{i} \cdot x_{i}-a_{i}-\frac{m_{i} h_{i} x_{i}^{2}}{2}\right)=0, i=1,2, \ldots, n
$$

We get

$$
x^{*}=\left[x_{1}^{*}, x_{2}^{*}, x_{n}^{*}\right], x_{i}^{*}=\frac{p_{i}}{h_{i} m_{i}}
$$

As an approximate solution of the problem we take the vector

$$
\hat{x}^{*}=\left[\hat{x}_{1}^{*}, \hat{x}_{2}^{*}, \ldots, \hat{x}_{n}^{*}\right]
$$

where

$$
\hat{x}_{i}^{*}=\operatorname{integ}\left(\frac{p_{i}}{h_{i} m_{i}}\right)
$$

\section{The Case of Discrete Distributions of the Demand of Multi Nomenclature Products}

In [19] the one-period multiproduct inventory control problem for discrete and continuous distributions for some fuzzy variables $D_{i}$. We will consider only the case of discrete variables possibility distributions $D_{i}$, to which it is easy to make a discrete probability distribution. As will be shown in the next section, from which it can directly receive already discrete, piecewise constant distribution function, which has the same form with arbitrary empirical distribution function can be approximated by [20] (Continuous) Hyper distribution function (the sum of a finite number of Erlanger distribution functions) a probability distribution for a discrete random variable sequence under consideration (in this case, the demand D), the corresponding discrete sequence STI observation times. Suppose that in the model (4) the demand $\mathrm{D}$ has the following distribution possibilities

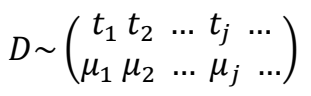

where $t_{1} \geq t_{2} \geq \cdots \geq t_{j} \geq \cdots$ is the ordered series of discrete values $t_{j}$ of the quantity $\mathrm{D}$ taken with the probabilistic (or probability) measure $\mu_{j}>0$, and

$$
\max _{1<j<+\infty} \mu_{j}=1 \text {. }
$$

As was shown in [19], under these conditions the expected value of $E[1 / D]$ will be equal to

$$
E\left[\frac{1}{D}\right]=\sum_{j=1}^{\infty} \frac{q_{j}}{t_{j}}
$$

where the weights $q_{j}$ are determined by the formula

$$
q_{j}=\frac{1}{2}\left(\max _{\tilde{\jmath} \leq j} \mu_{\tilde{\jmath}}-\max _{\tilde{j} \leq j-1} \mu_{\tilde{\jmath}}\right)+\frac{1}{2}\left(\sup _{\tilde{\jmath} \geq j} \mu_{\tilde{\jmath}}-\right.
$$

for any $j \geq 1 ; \mu_{0}=0$

The expected values of $E\left(1 / D_{i}\right)$ for the multi nomenclature problem are determined in a similar way:

$$
E\left[\frac{1}{D_{i}}\right]=\sum_{j=1}^{\infty} \frac{q_{i j}}{t_{i j}}
$$

Where $t_{i 1} \geq t_{i 2} \geq \cdots \geq t_{i j} \geq \cdots, \max _{1 \leq j<+\infty} \mu_{i j}=1 ; t_{i j}$ the ordered values of the demand $D_{i}$, taken with the possible measure $\mu_{\mathrm{ij}}$;

$$
q_{i, j}=\frac{1}{2}\left(\max _{\tilde{\jmath} \leq j} \mu_{i, \tilde{\jmath}}-\max _{\tilde{\jmath} \leq j-1} \mu_{i, \tilde{\jmath}}\right)+\frac{1}{2}\left(\sup _{\tilde{\jmath} \geq j} \mu_{i, \tilde{\jmath}}-\right.
$$

\section{Hypererlang Approximation of Arbitrary Distributions}

Let $\mathrm{X}$ be a nonnegative random variable (abbreviated to rv) with an arbitrary distribution function (abbreviated df) $F_{X}$. 
We are given an arbitrary number $\delta>0$. We divide the semi axis $[0, \infty)$ into half-open intervals $I_{k}=((k-1) \delta, k \delta], k \geq$ 1 , and choose a natural number $\mathrm{N}$ such that

$$
F_{X}((N-1) \cdot \delta) \geq 1-\delta
$$

We choose the points $x_{k} \in I_{k}, k=1, \ldots, N-1$ and $x_{N} \geq(N-1) \delta$.

Let $y_{k}=\lim _{x \rightarrow k \delta} F_{X}(x), k=1, \ldots, N-1$ We define a piecewise constant f.r. $\mathrm{F}_{\mathrm{X}}$ according to the following rule:

$$
F_{X}=\left\{\begin{array}{cc}
0, & x \leq x_{1} \\
y_{k}, & x_{k}<x \leq x_{k+1}, k=1, \ldots, N-1 \\
1, & x>x_{N}
\end{array}\right.
$$

We note that by rule (18) empirical distribution functions are constructed, in this case

$$
y_{k}=\frac{k}{N}
$$

For comparison d.f. $\mathrm{F}_{\mathrm{X}}(\mathrm{x})$ and $\mathrm{F}(\mathrm{x})$, we use the Levi metric [20]:

$$
\begin{aligned}
& L\left(F_{X}, F\right)=\inf \left\{\varepsilon>0: F_{X}(x-\varepsilon)-\varepsilon \leq F_{X}(x+\varepsilon)+\right. \\
& \varepsilon\} \forall x \in \mathfrak{R}^{1}
\end{aligned}
$$

The meaning of the Levi metric is very transparent - this is the side of the maximal square inscribed between the graphs of the d.f $F_{X}(x)$ and $F(x)$.

By construction d.f $F$ we have

$$
L\left(F_{X}, F\right) \leq \delta
$$

and

$$
F(x)=\sum_{k=1}^{N} p_{k} \cdot D g_{x_{k}}(x)
$$

Where $\quad p_{1}=y_{1}, p_{k}=g_{k}-y_{k-1}, 1<k \leq N-1, p_{N}=$ $1-y_{N-1}, D g_{x_{k}}(x)$ is the distribution, degenerate At the point $x_{k}$, i.e. $\left.D g_{x_{k}}(x)\right|_{x=x_{k}}=x_{k}$.

We approximate each of the degenerate distributions $D g_{x_{k}}(x)$ (x) by the Erlang distribution $E r_{n_{k}}^{\lambda_{k}}$. The Erlanger distribution is defined as follows [20].

Let $E_{i}=E_{i}(1)$ be a sequence of independent identically distributed random variables (abbreviated as nos) having an exponential distribution with a unit mean: $\left(E_{1}<x\right)=1-$ $e^{-x}$ We fix the number $\tau>0$ (for example, $\tau=1$ ) and determine for each $n \geq 1$ a random variable

$$
S_{n}^{\tau}:=\frac{\tau}{n} \sum_{i=1}^{n} E_{i}
$$

with the Erlanger distribution of order $n$ :

$$
P\left(S_{n}^{\tau}<x\right)=\operatorname{Er}_{n}^{\lambda}(n)=1-\sum_{i=0}^{n-1} \frac{(\lambda x)^{i}}{i !} e^{-\lambda x}
$$

where: $\lambda=n / \tau$.

It is well known that $S_{n}^{\tau} \underset{n \rightarrow \infty}{\longrightarrow} \tau$ with probability 1 , or, equivalently,

$$
E r_{n}^{\lambda}(x) \underset{n \rightarrow \infty}{\longrightarrow} D g_{\tau}(x)
$$

Where $D g_{\tau}(x)$ is the distribution degenerate at the point $\tau$. The limiting relation (25) is a consequence of equality (23) and the law of large numbers.

The distribution function Eh $(\cdot)$ is said to be hyperherlangian if it has the representation:

$$
E h(x)=\sum_{k=1}^{N} p_{k} E r_{n_{k}}^{\lambda_{k}}(x)
$$

where: $N<\infty, \sum_{k=1}^{N} p_{k}=1, p_{k}>0, \lambda_{k}>0, k=1, \ldots, N$.

As was shown in [20], for an arbitrary distribution $F_{X}$ of the form (18) and the hyper-Erlanger distribution (26) approximating it with coefficients $p_{\mathrm{k}}$ from (22), the accuracy of estimating the approximation in the Levi metric is described by the inequality

$$
L\left(F_{X}, E h\right) \leq \delta+\max _{1 \leq k \leq N} p_{k} \varepsilon_{k}
$$

Where $\delta$ is an arbitrary number; the number $\mathrm{N}$ satisfies condition (17); And the quantities $\varepsilon_{\mathrm{k}}$ are given by the righthand sides of the inequalities

$$
L\left(S_{n}^{\tau}, \tau\right)=\max \left(\tau \sqrt{\frac{\ln n}{n}}, \frac{1,48}{\sqrt{n}}\right)
$$

c $n=n_{k}, k=1,2, \ldots, N$.

The estimate (27) is universal in the sense that it is valid for arbitrary $\varphi . F(x)$ of the form (18).

Let the components $\mathrm{D}_{\mathrm{i}}$ of the vector $D=\left(D_{1}, \ldots, D_{n}\right)$ be described by empirical probability distribution functions

$$
F_{i}(x)=\left\{\begin{array}{cc}
0, & x \leq x_{i, 1} \\
y_{i, k_{i},}, & x_{i, k_{i}}<x \leq x_{i, k_{i}+1}, k_{i}=1, \ldots, N_{0, i}-1 \\
1, & x>x_{i, N_{0, i}}
\end{array}\right.
$$

where in

$$
\lim _{x \rightarrow x_{x_{k_{i}+1}}} F_{i}(x)=y_{i, k_{i}}=\frac{k_{i}+\left(r_{i, k_{i}}-1\right)}{N_{0, i}}
$$

where: $r_{i, k_{i}}$ multiplicity $x_{i, k_{i}}$ in the time series $\left\{x_{i, k_{i}}\right\}\left\{\mathrm{k}_{\mathrm{i}}\right.$ $\left.=1, . ., N_{0, i}\right\}$

We choose a natural number $N_{i}$ such that the number $\delta_{i}=\left(x_{i, N_{0, i}}-x_{i, 1}\right) / N_{i}$ satisfies the condition

$$
\begin{gathered}
\delta_{i}<\min _{k_{i}=1, \ldots, N_{0}, i-1}\left(x_{i . k_{i+1}}-x_{i, k_{i}}\right) \\
y_{i, N_{0, i}} \geq 1-\delta_{i}
\end{gathered}
$$

We partition the half-interval $\left(x_{i, 1}, x_{i, N_{0, i}}\right]$ into halfintervals of length $\delta_{\mathrm{i}}$ :

$$
\left(\hat{x}_{i, 1}, \hat{x}_{i, 1}+\delta\right],\left(\hat{x}_{i, 1}+\delta, \hat{x}_{i, 1}+2 \delta\right), \ldots,\left(\hat{x}_{i, 1}+\left(\hat{k}_{i}-1\right) \delta, \hat{x}_{i, 1}+\hat{k}_{i} \delta\right],
$$

$\left(\hat{k}_{i}=1,2, \ldots, N_{i}\right), \hat{x}_{i, 1}=x_{i, 1}, \hat{x}_{i, N_{i}}=x_{i, N_{0, i}}=\hat{x}_{i, 1}+N_{i} \cdot \delta$.

We denote by $I_{i, k}=\left(\hat{x}_{i, k-1}, \hat{x}_{i, k}\right), \hat{x}_{i, 1}=x_{i, 1}+k \cdot \delta$.

It's obvious that

$$
\hat{x}_{i, N_{i}}>(N-1) \delta
$$

As the distribution function $F_{X_{i}}(x)$ we define a piecewise 
constant function

$$
F_{X_{i}}(x)=\left\{\begin{array}{cc}
0, & x \leq \hat{x}_{1}, \\
\hat{y}_{i, \hat{k}_{i}}, & \hat{x}_{i, \hat{k}_{i}}<x \leq \hat{x}_{i, \hat{k}_{i}+1}, \hat{k}_{i}=1, \ldots, N_{i} \\
1, & x>\hat{x}_{i, N_{i}},
\end{array}\right.
$$

where

$$
\hat{y}_{i, \hat{k}_{i}}=y_{i, k_{i}} \text { at } x_{i, k_{i}}<\hat{x}_{i, \hat{k}_{i}+1} \leq x_{i, k_{i}+1} .
$$

The distribution function $F_{X_{i}}(x)$ will be approximated by the hyper-Erlanger distribution

$$
\begin{gathered}
E h_{i}(x)=\sum_{\hat{k}_{i}}^{N_{i}} p_{i, \hat{k}_{i}}(x) \operatorname{Er}_{n_{i, \widehat{k}_{i}}}^{\lambda_{i, \widehat{k}_{i}}}(x) \\
\mathrm{c} \tau=1, \lambda_{i, k}=n_{i, \hat{k}_{i}}, p_{i, 1}=\hat{y}_{i, 1}, p_{i, \hat{k}_{i}}=\hat{y}_{i, \hat{k}_{i}}-\hat{y}_{i, \hat{k}_{i}-1}, 1 \leq \\
\hat{k}_{i} \leq N_{i}-1, \\
p_{i, N_{i}}=1-y_{N_{i}-1} .
\end{gathered}
$$

According to (27)

$$
L\left(F_{X_{i}}, E h_{i}\right) \leq \delta_{i}+\max _{1 \leq \hat{k}_{i} \leq N_{i}} p_{i, \hat{k}_{i}} \cdot \varepsilon_{i, \hat{k}_{i}}
$$

where

$$
\varepsilon_{i, \widehat{k}_{i}}=\max \left(\sqrt{\frac{\ln n_{i, \widehat{k}_{i}}}{n_{i, \widehat{k}_{i}}}}, \frac{1,48}{\sqrt{n_{i, \widehat{k}_{i}}}}\right)
$$

Let $\delta$ be a given accuracy of the estimate $L\left(F_{X_{i}}, E h_{i}\right)$. We choose $\delta_{i}$, satisfying, along with conditions (31), (32), the condition

$$
\delta_{i} \leq \frac{\delta}{2}
$$

Then we can choose $n_{i, \hat{k}_{i}}$ such that

$$
\max _{1 \leq \hat{k}_{i} \leq N_{i}} p_{i, \hat{k}_{i}} \cdot \varepsilon_{i, \hat{k}_{i}} \leq \frac{\delta}{2}
$$

In conjunction with (38) providing an estimate

$$
L\left(F_{X_{i}}, E h_{i}\right) \leq \delta
$$

\section{Calculation of the Solution of the Task of Managing Multi Nomenclature Reserves}

According to formula (10), to find the solution of problem (8) it is sufficient to calculate the quantities $E\left(1 / D_{i}\right)$, where

$$
D \sim\left(\begin{array}{cccc}
t_{i, 1} & t_{i, 2} & \ldots & t_{i, N_{i}} \\
\mu_{i, 1} & \mu_{i, 2} & \ldots & \mu_{i, N_{i}}
\end{array}\right)
$$

Here, $t_{i, 1} \geq t_{i, 2} \geq \cdots \geq t_{i, N_{i}}$ are the ordered values of $t_{i, \hat{k}_{i}}$ of demand $\mathrm{D}_{\mathrm{i}}$, ordered in decreasing order, with probability measure $\mu_{i, \hat{k}_{i}}$. Since the distribution function $E h_{i}(x)$ is differentiable with respect to $x$. Then the probability measure $\mu_{i}(x)$ of the demand $\mathrm{x}$ values $\mathrm{D}_{\mathrm{i}}$ is expressed by the formula

$$
\mu_{i}(x)=\frac{d}{d x} E h_{i}(x)
$$

We denote by $t_{i, 1}=\hat{x}_{i, N_{i}}, t_{i, 2}=\hat{x}_{i, N_{i}-1}, \ldots, t_{i, N_{i}-1}=\hat{x}_{i, 2}$.

The approximate value for $\mu_{i, \hat{k}_{i}}=\mu_{i}\left(t_{i, \hat{k}_{i}}\right)$ can be determined by the formula

$$
\mu_{i, \hat{k}_{i}}=\left(E h_{X_{i}}\left(\hat{x}_{i, \hat{k}_{i}}+\alpha\right)-E h_{X_{i}}\left(\hat{x}_{i, \hat{k}_{i}}\right)\right) \cdot \alpha
$$

However, since $E h_{i}(x)$ is only an approximate value of the function $F_{X}(x)$ with accuracy $\delta$, the classical problem of approximate calculation of the derivative $z=\frac{d u(x)}{d x}$ with respect to approximate (in the metric of $\mathrm{C}$ continuous functions) is incorrect and can be solved with the aid of Regulatory operator [23]

$$
R(u, \alpha)=\frac{u(x+\alpha)-u(x)}{\alpha}
$$

In fact, let $u(x)=E h_{X_{i}}$ and instead of the exact values of the functions $\mathrm{u}(\mathrm{x})$ we have approximate values $u_{\delta}(x)=$ $u(x)+v(x)$, where $v(x) \leq \delta$ for $\mathrm{X} \in(\mathrm{a}, \mathrm{b})$. In our case, $(a, b)=\left(\hat{x}_{i, 1}, \hat{x}_{i, N_{i}}\right)$ and the accuracy of (40) implies the accuracy $\delta$ of estimating the approximation of the distribution function $F_{X_{i}}(x)$ by the hyper-Erlanger The distribution function $E h_{X_{i}}(x)$.

Then

$$
R\left(u_{\delta}, \alpha\right)=\frac{u(x+\alpha)-u(x)}{\alpha}+\frac{v(x+\alpha)-v(x)}{\alpha}
$$

As $\alpha \rightarrow 0$, the first fraction in (44) tends to the derivative $\mathrm{du} / \mathrm{dx}$. If we take $\alpha=\delta / \eta(\delta)$, where $\eta \rightarrow 0$ as $\delta \rightarrow 0$, then $2 \delta /(\alpha=2 \eta(\delta) \rightarrow 0)$ as $\delta \rightarrow 0$ and, consequently, for $\alpha=\alpha_{1}(\delta)=\frac{\delta}{\eta(\delta)}$ we have

$$
\left|\frac{v(x+\alpha)-v(x)}{\alpha}\right| \leq \frac{2 \delta}{\alpha}
$$

And therefore $R\left(u_{\delta}, \alpha_{1}(\delta)\right) \rightarrow \frac{d u}{d x}$ It suffices to take $\eta(\delta)=\delta^{1-\varepsilon} \quad, \quad\left(\begin{array}{lll}0 & <\varepsilon & <1\end{array}\right) \quad$ then $\alpha=\delta^{\varepsilon} \quad$ and $2 \delta / \alpha=2 \delta^{1-\varepsilon} \rightarrow 0$ as $\delta \rightarrow 0$.

\section{Conclusion}

In conditions of competition in the market of motor transport services, ensuring maximum profit is one of the main tasks of managing multinational stocks of auto service enterprises using the logistics concept. Approximation of empirical distribution functions of the demand vector components allows us to calculate the corresponding density distribution of the values of the components of the demand vector and reduce the problem of determining the optimal stock size to the quadratic problem of conditional optimization. 


\section{References}

[1] Liners M. R., Firon H. E. Supply and inventory management. Logistics. - St. Petersburg: Victoria Plus LLC, 2002. - 768 p.

[2] Lukinsky VS And others. Logistics of motor transport. Concept, methods, models. - Moscow: Finance and Statistics, 2000.

[3] Shchetina VA, Lukinsky VS, Sergeev VI Supply of spare parts for road transport. - Moscow: Transport, 1988. - 109 p.

[4] Shcherbakov D. A. Logistical methods and models of the organization of service and supply management in the systems of firm car service. - Dis. Na syisk. uchen. steni can. Ekonom. nauk. - St. Petersburg, 2003. - 142s.

[5] Maslikov AI Methods and models of managing multinomenclature inventory in the distribution company. Dis. To the soot. Scientist. The degree of Cand. Sc. - St. Petersburg, 2009. - 172s.

[6] Tikhomirova AN, Sidorenko EV Mathematical Models and Methods in Logistics: Textbook. M.: NNIU MEPhI, 2010. 320 s.

[7] Bowersox D. J., Closs D. J. Logistics. Integrated supply chain. Moscow: ZAD Olimp-Business, 2008. - 632p.

[8] Kisel TR, Buiko LA A logistical approach to the management of an auto transport enterprise. // Bulletin of BNGU, 2006, №4, p. 64-70.

[9] Logistics: Textbook / Under the Red. BAAnikina. - Moscow: 2000. $-352 \mathrm{p}$.

[10] Models and methods of the theory of logistics, Ed. V. S. Lukinsky. - St. Petersburg: Peter, 2003. - 203 p.

[11] Mohd-LaiR N-A, Muhiddin F-A., Laudi S., Mohd-Tamiri F., ChuA B-L. The spare part inventory management system (SPIMS) for profound heritagy SDN BHD (PHSB): a case study on the EOQ technique//International Journal of research Engineering Technology, vol. 2, Issue 1, Jan 2014, 7-14.

[12] Ivanov DA Supply chain management. - SPb.: Publishing house of the Polytechnical University, 2009. - 660c.
[13] Chang, S. Y., and Yeh, T. Y. A two- echelon supply chaun of a returnable product with fuzzy demand //Applied Mathematical Modelling, vol. 37, no 6, pp. 4305-4315. 2013.

[14] Liu, B., and Liu Y. K. Expected value of fuzzy variable and fuzzy expected value models //IEEE Transaction on Fuzzy System, vol. 10, no 4, pp. 445-450, 2002.

[15] Shao, Z., and Ji, X. Fuzzy multi-product constraint newsboy problem //Applied Mathematics and Computation, vol. 180, no 1, pp. 7-15, 2006.

[16] Yaghin, R. G., Ghomi, S. M. T. F., and Torabi S. A. A hybrid credibility-based fuzzy multiple objective oprimization to differential pricing and inventory policies with arbitrage consideration //International Journal of System Science, vol. 46, no 14, pp. 2628-2639, 2015.

[17] Yao, J. S., Chen, M. S., and Lu, H. F. A fuzzy stochastic single-period model for cash management //European Journal of Operational Research, vol. 170, no 1, pp. 72-90, 2005.

[18] Borgonovo, E., and Elhafsi, M. Financial management in inventory problems: risk averse vs risk neutral policies //International Journal of Production Economics, vol. 118, no. 1, pp. 233-242, 2009.

[19] Li, Y.-N., Y. K. and Liu, Y. K. Oprimizing Fuzzy Multitem Single-period Inventory Problem under Risk-neutral Criterion //Journal of Umertain Systems, vol. 10, no. 2, pp. 130-141, 2016.

[20] Kalashnikov VV, Rachev S. T. Mathematical methods for constructing stochastic service models. - Moscow: Nauka, 1988. - 312p.

[21] Liu, Y. K., and Gao J. The independent of fuzzy variables with application to fuzzy random optimization //International Journal of Production Economics, vol. 81-82, pp. 315-384, 2003.

[22] Liu, J. K., and Liu, B. Expected value operator of random fuzzy variable and random fuzzy expected value models //International Journal of Uncertainty, Fuzzines and Knowledge-Based Systems, vol, 11, no. 2, pp. 195-215, 2003.

[23] Tikhonov AN, Arsen'ev V. Ya. - Moscow: Nauka, 1979. 285 p. 